\title{
RNA-Seq Analysis for Exploring the Pathogenesis of Retinitis Pigmentosa in P23H Knock-In Mice
}

\author{
Jiarui Li ${ }^{a, b, c}$ Wei Du ${ }^{a, b, c}$ Ningda Xua,b, c Tianchang Tao ${ }^{a, b, c}$ Xin Tang ${ }^{a, b, c}$ \\ Lvzhen Huang ${ }^{a, b}, c$
}

${ }^{a} E y e$ diseases and Optometry Institute, Department of Ophthalmology, Peking University People's Hospital, Beijing, China; ${ }^{b}$ Beijing Key Laboratory of Diagnosis and Therapy of Retinal and Choroid Diseases, Beijing, China; ${ }^{\circ}$ College of Optometry, Peking University Health Science Center, Beijing, China

\section{Keywords}

Retinitis pigmentosa · ER stress · Pyroptosis · RNA-seq ·

Proline 23 substituted by histidine

\begin{abstract}
Introduction: Mechanisms contributing to the progression of autosomal dominant retinitis pigmentosa (adRP) due to the $\mathrm{P} 23 \mathrm{H}$ rhodopsin mutation are complex and diverse. Previous studies showed that mechanisms like endoplasmic reticulum (ER) stress, pyroptosis, and oxidative stress were involved in the pathogenesis of the disease. However, the roles and relationships of different mechanisms are not precisely known. In this study, we aimed to evaluate certain mechanisms and find novel genes involved in $\mathrm{P} 23 \mathrm{H}$-related adRP. Methods: Total RNA extracted at postnatal day (PN) 14, PN21, and PN35 was used for RNA sequencing. Gene Ontology and Kyoto Encyclopedia of Genes and Genomes functional enrichment analyses were conducted for RNA-seq data. Additionally, data from the clustered regularly interspaced short palindromic repeats (CRISPR) screening library and the RNA-seq data of several mechanisms were used for generating custom gene sets for gene set enrichment analysis (GSEA). Next, we obtained the intersection of the aforementioned gene sets and our RNA-seq data to identify candidate genes, which were verified using real-time quantita-
\end{abstract}

tive PCR (qPCR). Results: Functional enrichment analyses were consistent with disease phenotypes. All time points observed pyroptosis. In the results of GSEA, ER stress, pyroptosis, and oxidative stress were observed at PN14. ER stress and pyroptosis were shown on PN35. A total of 22 candidate genes were identified. The expression levels of selected genes verified by qPCR were concordant with the RNA-seq data. Conclusions: In our study, we conclude that pyroptosis and ER stress might play a central role in RP progression. We also identified differentially expressed gene clusters related to ER stress and pyroptosis, which deserve further study. These findings provide a novel perspective for the investigation of P23H-related adRP.

(C) 2021 The Author(s)

Published by S. Karger AG, Basel

\section{Introduction}

Retinitis pigmentosa (RP) is a group of inherited neurodegenerative retinopathies that are initially characterised by the dysfunction and death of rod photoreceptors with a progressive loss of the peripheral visual field. $\mathrm{Mu}$ tation in rhodopsin is a common cause of autosomal

Jiarui Li and Wei Du are co-first authors. karger@karger.com www.karger.com/ore

Karger $\stackrel{\text { ' }}{5}$

GOPEN ACCESS
(C) 2021 The Author(s)

Published by S. Karger AG, Basel

This is an Open Access article licensed under the Creative Commons Attribution-NonCommercial-4.0 International License (CC BY-NC) (http://www.karger.com/Services/OpenAccessLicense), applicable to the online version of the article only. Usage and distribution for commercial purposes requires written permission.
Correspondence to:

Lvzhen Huang, huanglvzhen@ 126.com 
dominant RP (adRP), whereas proline 23 substituted by histidine $(\mathrm{P} 23 \mathrm{H})$ rhodopsin mutation was the first identified and the most studied mutation [1]. The reported pathogenesis of adRP due to $\mathrm{P} 23 \mathrm{H}$ rhodopsin mutation is complicated. Research focusing on the role of endoplasmic reticulum (ER) stress and the unfolded protein response (UPR) in the death of rod photoreceptor cells identified gene alterations in inositol-requiring enzyme 1 (IRE1), activating transcription factor 6 (ATF6), and PKR-like ER kinase (PERK) pathways [2, 3]. Previous studies had reported that the $\mathrm{P} 23 \mathrm{H}$ rhodopsin mutation led to the misfolding of rod opsin and subsequently induced UPR and ER stress. If the UPR did not alleviate the degradation of misfolded protein, it would culminate in cell death [4, 5]. However, other shreds of evidence showed that pyroptosis, oxidative stress, autophagy, and calpain activation also contributed to the progression of RP [6-9]. Additionally, the ablation of the UPR-related gene Chop did not delay the dysfunction of photoreceptors. Thus, ER stress and UPR might regulate secondary cellular responses [10]. Additionally, microglia and Müller glial cells are also activated in retinal degeneration [11, 12]. The aforementioned pieces of information show that several mechanisms play an essential role in RP pathogenesis. However, the roles and relationships of the different mechanisms are still under debate.

Presently, research to explore the progression of adRP due to the $\mathrm{P} 23 \mathrm{H}$ rhodopsin mutation is mostly based on a single gene or simple pathway. Therapies used to improve visual function, such as drugs and gene therapy, were either designated to target a single gene or their mechanism of action [13]. Thus, a macro-perspective is necessary for a better understanding of RP progression.

Our study aimed to macroscopically evaluate the different mechanisms and identify crucial genes involved in the progression of adRP due to the $\mathrm{P} 23 \mathrm{H}$ rhodopsin mutation, hoping to provide valuable clues and insights for the advancement of the understanding of the $\mathrm{P} 23 \mathrm{H}$ rhodopsin mutation adRP.

\section{Materials and Methods}

\section{Animals}

The $\mathrm{P} 23 \mathrm{H}$ knock-in mice $\left(R h o^{P 23 H / P 23 H}\right)$ [14] were obtained from the Jackson Laboratory (017628; Jackson Laboratory, Bar Harbor, ME, USA) and C57BL/6J (B6J) serving as a control were purchased from Charles River (Charles River Laboratory Animal, Beijing, China). Two groups were bred separately to generate homozygous offspring for the following study. All mice were housed in the same animal room from the time of birth, considering environmental effects. This study adhered to the statement of the As- sociation for Research in Vision and Ophthalmology for the Use of Animals in Ophthalmic and Vision Research. Experimental procedures were approved by the Institutional Animal Care and Use Committee of Peking University People's Hospital (permit number: 2016PHC059). All animals were raised under the following conditions: room temperature, $55 \pm 5 \%$ humidity, and a 12/12-h artificial light/dark cycle.

\section{RNA Isolation}

Mice were euthanised at postnatal day (PN) 07, PN10, PN14, PN21, and PN35 by cervical dislocation while on anaesthetic overdose. Immediately after euthanasia, eyeballs and retinas were isolated. For RNA-seq, 6 retinas were used for 1 sample. For real-time quantitative PCR (qPCR), 1 retina was used per sample. The total RNA of the retina was isolated using the Monarch total RNA Miniprep Kit (New England Biolabs, \#T2010S). The total RNA of each sample was qualified and quantified using an Agilent 2100 Bioanalyzer (Agilent Technologies, Palo Alto, CA, USA), NanoDrop (Thermo Fisher Scientific Inc.).

\section{RNA Sequencing}

After the quality test, the total RNA from PN14, PN21, and PN35 of $R h o^{P 23 H / P 23 H}$ and B6J mice was used for next-generation sequencing library preparations, conducted according to the manufacturer's protocol. The rRNA was separated from the total RNA using the rRNA removal kit. The obtained RNA was then fragmented and reverse-transcribed. The first-strand cDNA was synthesised using ProtoScript II Reverse Transcriptase with random primers and actinomycin D. The second-strand cDNA was synthesised using the Second Strand Synthesis Enzyme Mix (include dACG-TP/dUTP). The double-stranded cDNA purified by beads was then treated with the End Prep Enzyme Mix to repair both ends and add a dA-tailing in 1 reaction, followed by T-A ligation to add adaptors to both ends. Size selection of adaptor-ligated DNA was then performed using beads, and fragments of $\sim 420$ bp (with the approximate insert size of $300 \mathrm{bp}$ ) were recovered. The dUTP-marked second strand was digested with a Uracil-Specific Excision Reagent enzyme. PCR then amplified each sample for 13 cycles using the P5 and P7 primers, both primers carrying sequences that can anneal with flow cells to perform bridge PCR and P7 primer carrying a 6-base index allowing for multiplexing. The PCR products were cleaned up using beads, validated using a Qsep100 (Bioptic, Taiwan, China), and quantified by a Qubit 3.0 Fluorometer (Invitrogen, Carlsbad, CA, USA). Libraries with different indices were multiplexed and loaded on an Illumina HiSeq instrument according to the manufacturer's protocols (Illumina, San Diego, CA, USA). Sequencing was conducted using a $2 \times 150$ paired-end configuration. Image analysis and base calling were performed by HiSeq Control Software + OLB + GAPipeline-1.6 (Illumina) on the HiSeq instrument.

\section{RNA-Seq Data Analysis}

The high-quality clean data were prepared by Cutadapt (V1.9.1) and then aligned to the reference genome (GRCm38) via Hisat2 (v2.0.1). Next, feature counts (v2.0.0) [15] were used for estimating gene expression levels from the clean data. Counts were input into GFOLD (v1.1.4) [16] for differential expression analysis. GFOLD is a reliable tool for obtaining RNA-seq data without biological replicates. According to the manufacturer's protocol, the GFOLD value was approximately equal to a $\log 2$-fold change. A gene with a zero GFOLD value should not be considered a differentially ex- 
Table 1. Primers for real-time qPCR

\begin{tabular}{|c|c|c|c|c|}
\hline $\begin{array}{l}\text { Gene } \\
\text { symbol }\end{array}$ & Ensembl ID & Sequence $\left(5^{\prime}>3^{\prime}\right)$ & $\begin{array}{l}\text { Amplicon } \\
\text { size, bp }\end{array}$ & Sources \\
\hline Rho & ENSMUSG00000030324 & Fp: CССТTCTCCAACGTCACAGGRp: TGAGGAAGTTGATGGGGAAGC & 126 & {$[21]$} \\
\hline Kcnj14 & ENSMUSG00000058743 & Fp: GCAATGTGCGCTTCGTGAATCRp: CCACTTGCGAGAAACAGGGA & 219 & $\begin{array}{l}\text { Primer bank ID } \\
22122399 \mathrm{a} 1\end{array}$ \\
\hline$C 4 b$ & ENSMUSG00000073418 & Fp: GCCATCCACAGAGAAGAGTTAGRp: TCAGGGTAGGAGCCAAATAGA & 256 & {$[23]$} \\
\hline Gapdh & ENSMUSG00000057666 & Fp: AACTTTGGCATTGTGGAAGGRp: GGATGCAGGGATGATGTTCT & 132 & {$[24]$} \\
\hline
\end{tabular}

Rho, Rhodopsin; Gnatl, G protein subunit alpha transducin 1; Kcnj14, potassium inwardly rectifying channel subfamily J member 14; Ifi44, interferoninduced protein 44; $C 4 b$, complement c4b; Gapdh, glyceraldehyde-3-phosphate dehydrogenase; Fp, forward primer; Rp, reverse primer; qPCR, quantitative PCR.

pressed gene (DEG). The STRING database (https://string-db.org/ cgi/input.pl) was used to execute GO (Gene Ontology) and KEGG (Kyoto Encyclopedia of Genes and Genomes) functional enrichment analysis [17]. The pre-ranked custom gene sets of the clustered regularly interspaced short palindromic repeats (CRISPR) screening library and RNA-seq data were entered into clusterProfiler (v3.14.3) [18] for gene set enrichment analysis (GSEA). It should be noted that we could access only upregulated genes in the RNA-seq data of pyroptosis; thus, for all the RNA-seq data, we used upregulated genes to perform GSEA and the following analysis. For the CRISPR screening library and RNA-seq data from humans, the R package biomaRt (v2.42.0) [19] was used to transform human gene IDs into mouse gene IDs.

\section{Real-Time $q P C R$}

A total of $2 \mu \mathrm{g}$ RNA was used to generate cDNA with the ReverTra Ace ${ }^{\circledR}$ qPCR RT Master Mix (Toyobo Co., Ltd., Osaka, Japan, Code No. FSQ-201). Real-time qPCR were conducted using SYBR ${ }^{\circledR}$ Green Real-time PCR Master Mix (Toyobo Co., Ltd., Osaka, Japan, Code No. QPK-201) with an Applied Biosystems ${ }^{\text {TM }} 7500$ Real-Time PCR System (Applied Biosystems, Waltham, MA, USA). The experiments were repeated using at least 3 biological and 3 technical replicates. Analysing relative mRNA expression levels by the $2^{-\triangle \mathrm{CT}}$ methods using Gapdh as endogenous control, the fold change in expression levels was calculated by a previously reported protocol [20]. Table 1 lists all primers [21-24].

\section{Tissue Staining}

After removal of the cornea, lens, and vitreous, the eyecups at PN14, PN21, and PN35 were fixed with 4\% paraformaldehyde for $24 \mathrm{~h}$ and then embedded in paraffin and sectioned. The sections were subjected to hematoxylin and eosin staining according to the standard procedure. Four points at the inferior and superior retina, respectively, were measured for the statistical analysis of the outer nuclear layers.

\section{Flash Electroretinogram Recording}

According to the standards of the International Society for Clinical Electrophysiology of Vision, full-field flash ERG was recorded by an ERG recording system (RETIport, Roland Consult, Germa- ny). All procedures were performed in a dark room under dim redlight illumination (>650 nm). B6J and $R h o^{P 23 H / P 23 H}$ mice were darkadapted overnight. The pupil was dilated 3 times for $5 \mathrm{~min}$ with compound tropicamide eye drops containing $0.5 \%$ tropicamide and $0.5 \%$ phenylephrine (Santen Pharmaceutical Co. Ltd, Japan). After mydriasis, the mice were anaesthetised by intraperitoneal phenobarbital $(50 \mathrm{mg} / \mathrm{kg}$ ) and xylazine $(5 \mathrm{mg} / \mathrm{kg})$ under a dim redlight illumination. Then the mice were placed on the thermal platform and maintained at $37^{\circ} \mathrm{C}$ in the prone position. Stainless steel subdermal needle electrodes were placed at the tail (ground electrode) and under individual eyelids as reference electrodes. Recording gold electrodes were placed directly onto the cornea, and $0.1 \%$ sodium hyaluronate eye drops (Santen Pharmaceutical Co., Ltd., Japan) were used to keep the cornea moist. Under dark-adapted conditions, flash ERG recordings were obtained simultaneously from both eyes, with the intensity of white light stimulation for scotopic ERG initially set to $0.001 \mathrm{~cd} \cdot \mathrm{s} / \mathrm{m}^{2}$. The stimulus intensity was then increased to $0.003,0.3$, and $3 \mathrm{~cd} \cdot \mathrm{s} / \mathrm{m}^{2}$. The mice were then light-adapted in a photopic background $\left(25 \mathrm{~cd} \cdot \mathrm{s} / \mathrm{m}^{2}\right)$ for $15 \mathrm{~min}$. The stimulus intensity for photopic ERG was initially $1 \mathrm{~cd} \cdot \mathrm{s} / \mathrm{m}^{2}$ and then increased to 3 and $10 \mathrm{~cd} \cdot \mathrm{s} / \mathrm{m}^{2}$. Each recording was averaged 3 times at the aforementioned luminance levels.

\section{Statistical Analysis}

We performed independent Student $t$-tests to reveal the differences between groups at the same time points, after conducting the Shapiro-Wilk normality test and Levene's test for homogeneity of variances. If data failed to pass the normality test and the variance homogeneity test, then comparisons were made using the MannWhitney nonparametric test. A $p$ value of $<0.05$ was considered a significant difference.

\section{Results}

\section{RNA Sequencing Results}

We chose the time points for RNA-seq from the previously reported $\mathrm{RP}$ progress curve of $\mathrm{Rh} \mathrm{o}^{\mathrm{P2} H / \mathrm{P} 23 \mathrm{H}}$ mice 
Table 2. Number of DEGs in RNA-seq

\begin{tabular}{lll}
\hline Day & $\begin{array}{l}\text { Upregulated } \\
\text { genes }\end{array}$ & $\begin{array}{l}\text { Downregulated } \\
\text { genes }\end{array}$ \\
\hline PN14 & 32 & 71 \\
PN21 & 19 & 262 \\
PN35 & 14 & 365 \\
\hline
\end{tabular}

PN, postnatal; DEG, differentially expressed gene.

[25]. RNA-seq identified DEGs (GFOLD $>1.5$ ) on the retinas of $R h o^{P 23 H / P 23 H}$ mice versus C57BL/6J (B6J) mice. Table 2 summarises the data. From PN14 to PN35, the number of downregulated DEGs increases with RP progression. To obtain more bioinformatic signatures about DEGs, we next performed GO and KEGG pathway enrichment analysis (shown in Fig. 1; see online suppl. Table 1; for all online suppl. material, see www.karger.com/ doi/10.1159/000515727 provides complete information). We found only 33 upregulated GO terms significantly enriched at PN14. Figure 1a shows the top 20 GO terms. Some GO terms related to intermediate filament cytoskeleton and immune response are enriched. However, strikingly, we did not find a clear enrichment in cell death-related functions. A total of 89 and $80 \mathrm{GO}$ terms were identified at PN21 and PN35, respectively, and all the top downregulated GO terms enriched are associated with phototransduction and the structure of photoreceptors (shown in Fig. 1b, c). The results of the KEGG pathway analysis also show the phototransduction is the most significantly downregulated pathway at PN21 and PN35 but not PN14 (Fig. 1d). Taken together, we consider that PN21 and PN35 are in the advanced stage of rod photoreceptor death because of a significant enrichment of the visual pathway, but the aforementioned results do not give us sufficient evidence on mechanisms of RP progression.

\section{Using the GSEA Algorithm to Evaluate Possible Mechanisms}

As sufficient evidence for the mechanisms was not shown in the enrichment analysis and previous knowledge on enrichment analysis could limit our research, we browsed reported studies that contained genomic library or RNA-seq data and screened them for the following analysis (brief descriptions are shown in Table 3 [26-34]; details are in online suppl. Table 2). A total of 9 studies contained 4 main mechanisms, the size, and effect of them based on their screening criteria. Table 4 and Figure

Exploring the Pathogenesis of $\mathrm{P} 23 \mathrm{H}-$

Related Retinitis Pigmentosa by RNA-Seq
2 show the significant results of GSEA (complete data are in online suppl. Table 3). A normalised enrichment score $(\mathrm{NES})>0$ indicates upregulation, whereas a NES $<0$ indicates downregulation (the larger the number, the greater the influence). The upregulation of pyroptosis is observed at all time points (Fig. 2b, e, f). As expected, the upregulation of ER stress appears in PN14 (NES = 2.4171), and the downregulation of protectors of tunicamycin-induced ER stress is shown in PN35 (NES $=-1.3101$ ) (Fig. 2a, g). ER stress, pyroptosis (one is an RNA-seq gene set and another is a CRISPR screening library data gene set), and oxidative stress can be observed in PN14. Based on the previous GSEA results, we consider pyroptosis and ER stress as the main mechanisms involved in RP progression.

\section{Intersection with Custom Gene Sets Data for}

Predicting Critical Genes Involved RP Progression

After the evaluation of possible mechanisms, we intersected the data from the 9 aforementioned studies with our RNA-seq data. Bubble plots were used for displaying the primary outcomes. Most of the gene sets contained DEGs, except for the CRISPR screening library of oxidative stress and the RNA-seq data of oxidative stress (shown in Fig. 3a, b). A total of 22 genes labelled with their gene symbol were reported in the results. Among them, 10 genes were upregulated, while 12 genes were downregulated. Kcnj14 was the most significantly downregulated gene. Additionally, 3 genes (Ifi44, C4b, and Ifit1) appeared simultaneously in 2 gene sets from different mechanisms. In particular, Ifi44 and $C 4 b$ were significantly upregulated in at least 2 time points, so we suppose that these 2 genes play a role in the progression of RP.

\section{Validation of the Expression Levels of Selected Genes}

by Real-Time qPCR

Based on the previous analysis, we selected Ifi44 and $C 4 b$ as candidate genes for validating their expression levels. We included Kcnj14 in our analysis as it was among the most significantly downregulated genes in the bubble plots. Rhodopsin (Rho) and rod photoreceptor-specific transducin a-subunit (Gnat1) were used as reference genes for normal retinal development and the death of rod photoreceptors. As indicated in Figure 4, we validated the expression levels of 5 genes at 5 different time points (PN07, PN10, PN14, PN21, and PN35) to have a better understanding of the changing trend in these genes over time. We considered expression levels at PN07 as the baseline. The experiment shows that the mRNA level of Rho is considerably lower than that of the control group 


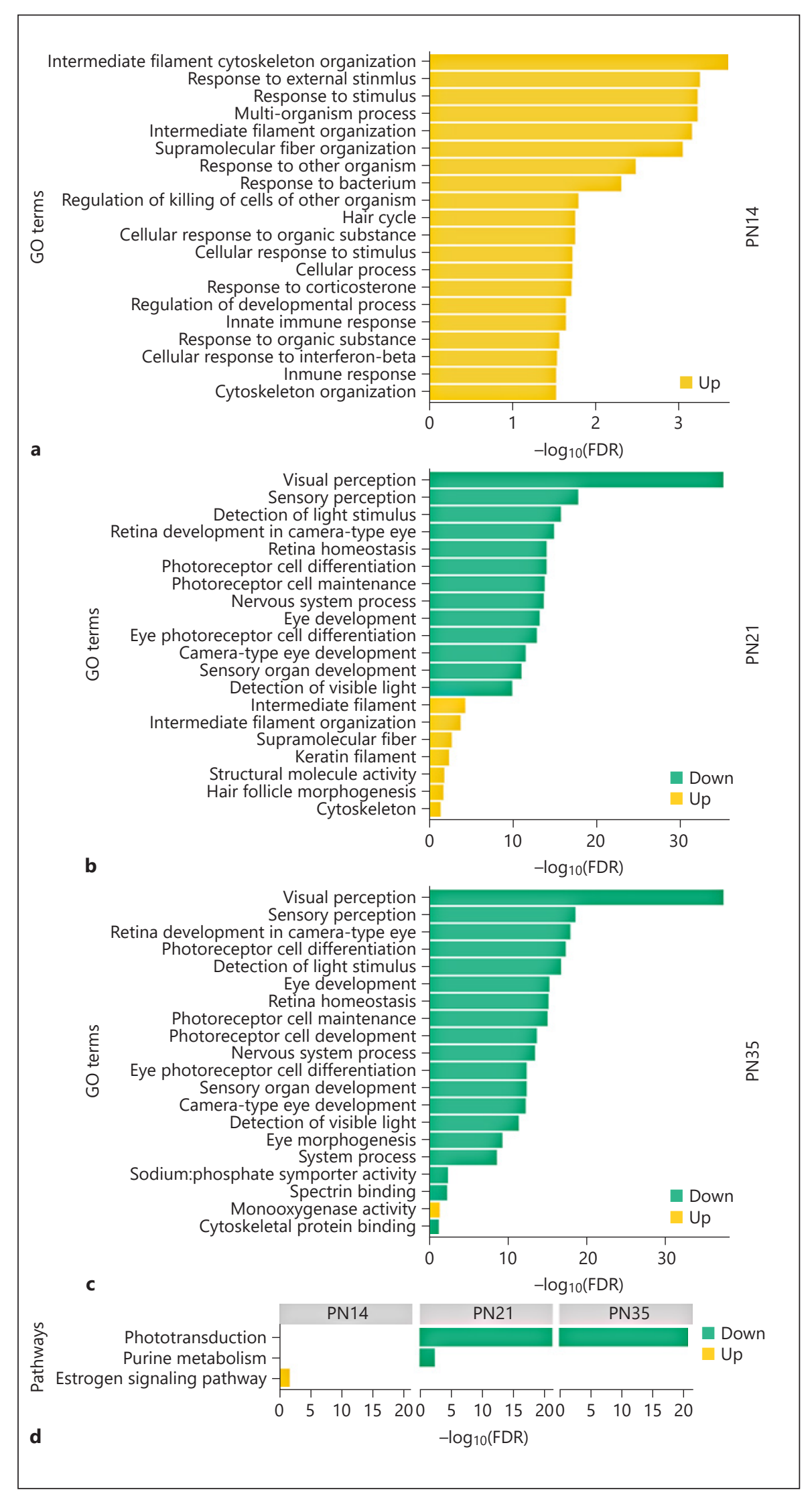

Fig. 1. GO and KEGG analysis of DEGs. a Top $20 \mathrm{GO}$ terms for DEGs specific to PN14. b Top 20 GO terms for DEGs specific to PN21. c Top 20 GO terms for DEGs specific to PN35. The screening criterion for GO analysis was $p$ adjust $<0.05$. d KEGG analysis of DEGs in PN14, PN21, and PN35 (FDR <0.05). Upregulated GO terms and pathways fill with yellow; downregulated $\mathrm{GO}$ terms and pathways fill with green; GO, Gene Ontology; KEGG, Kyoto Encyclopedia of Genes and Genomes (http://www.genome.jp/kegg/); DEGs, differentially expressed genes; PN, postnatal; FDR, false discovery rate. 
after PN10, whereas the Gnat1 level is significantly lower than that of the control group after PN14 (Fig. 4a, b). Additionally, the mRNA levels of Rho and Gnat1 increased with time in the B6J mice group, while those of Rho ${ }^{P 23 H / P 23 H}$ decreased after PN14 (Fig. 4a, b). The expression levels of Ifi44, C4b, and Kcnj14 show apparent differences when compared to B6J (Fig. 4c-e. Moreover, the correlation analysis of the log2 fold change between RNA-seq and qPCR data of these 5 genes indicates a high correlation $\left(R^{2}=0.9691\right.$, Fig. $\left.4 \mathrm{f}\right)$. According to the previous results, we conclude that the functional roles of Ifi44, $C 4 b$, and Kcnj14 deserve further study.

\section{Discussion/Conclusion}

Presently, the mechanisms underlying RP pathogenesis due to $\mathrm{P} 23 \mathrm{H}$ opsin mutations are sophisticated. Thus, it is imperative to explore its pathogenesis from a macro-perspective. We used RNA-seq for identifying DEGs at 3 different time points in the retinas of $R h o^{P 23 H / P 23 H}$ mice, using B6J mice as a control and obtaining results associated with this disease phenotype. As shown in online suppl. Fig. 1, the outer nuclear layer of $R h o^{P 23 H / P 23 H}$ mice is significantly thinner than that of B6J mice at PN14, PN21, and PN35. By PN35, the photoreceptor nuclei remained less than a

Table 3. Descriptions of CRISPR screening library data and RNA-seq data that used to perform GSEA and intersection

\begin{tabular}{|c|c|c|c|c|c|}
\hline Classification & Mechanisms & Species & Size & Effect & Library \\
\hline \multirow[t]{6}{*}{ CRISPR screening library } & $\mathrm{H}_{2} \mathrm{O}_{2}$-induced oxidative stress [26] & Human & 174 & Sensitisers (53)/protectors (121) & CRISPR/Cas9 knockout library (self-design) \\
\hline & Tunicamycin-induces ER stress [27] & Human & 422 & Sensitisers (133)/protectors (289) & AVANA library \\
\hline & CHOP-related ER stress [28] & Human & 271 & Protectors & Human GeCKOv2 library \\
\hline & NRF2-related cellular homeostasis [29] & Human & 235 & Protectors & Human activity-optimised CRISPR knockout library \\
\hline & Caspase 11-dependent pyroptosis [30] & Mouse & 100 & Sensitisers & CRISPR-Cas9 genome-wide knockout library \\
\hline & NLRP1B-mediated pyroptosis [31] & Mouse & 268 & Sensitisers & Mouse GeCKO2-vector system sgRNA pool B library \\
\hline \multirow[t]{3}{*}{ RNA-seq } & Pyroptosis [32] & Mouse & 114 & Upregulation & - \\
\hline & ER stress [33] & Mouse & 770 & Upregulation & - \\
\hline & Oxidative stress [34] & Human & 22 & Upregulation & - \\
\hline
\end{tabular}

Size: the number of gene hits or the DEGs of these mechanisms. ER stress, endoplasmic reticulum stress; NLRP1B, nucleotide-binding domain leucine-rich repeat pyrin domain-containing 1B; CRISPR, clustered regularly interspaced short palindromic repeats; GSEA, gene set enrichment analysis; DEG, differentially expressed gene.

Table 4. Significant results of GSEA

\begin{tabular}{|c|c|c|c|c|c|c|}
\hline Day & Mechanisms & Set size & ES & NES & $p$ value & $\begin{array}{l}q \text { value } \\
(\text { cut-off }=25 \%)\end{array}$ \\
\hline \multirow{3}{*}{ PN14 } & RNA-seq: ER stress & 311 & 0.3565 & 1.4685 & 0.0014 & 0.0017 \\
\hline & CRISPR library: caspase 11-dependent pyroptosis & 30 & 0.5203 & 1.4630 & 0.0373 & 0.0513 \\
\hline & CRISPR library: sensitisers of $\mathrm{H}_{2} \mathrm{O}_{2}$-induced oxidative stress & 24 & 0.5394 & 1.4456 & 0.0487 & 0.0513 \\
\hline \multirow[t]{2}{*}{ PN35 } & RNA-seq: pyroptosis & 49 & 0.5315 & 1.9420 & 0.0087 & 0.0183 \\
\hline & CRISPR library: protectors of tunicamycin-induced ER stress & 109 & -0.4659 & -1.3101 & 0.0608 & 0.2430 \\
\hline
\end{tabular}

Set size: the number of genes can be found in our RNA-seq data. ES, enrichment score; NES, normalised enrichment score; ER stress, endoplasmic reticulum stress; PN, postnatal; CRISPR, clustered regularly interspaced short palindromic repeats; GSEA, gene set enrichment analysis.

Fig. 2. GSEA plots of custom gene sets correspond to Table 4. a-d GSEA plots of PN14. Upregulation of ER stress in PN14 $(\mathrm{NES}=1.4685, p$ value $=0.0014)(\mathbf{a})$; upregulation of pyroptosis in PN14 $($ NES $=2.4171, p$ value $=0.0017)(\mathbf{b})$; upregulation of caspase 11 dependent pyroptosis in PN14 $(\mathrm{NES}=1.4630, p$ value $=0.0373)$ (c); upregulation of sensitisers of $\mathrm{H}_{2} \mathrm{O}_{2}$-induced oxidative stress in PN14 $($ NES $=1.4456, p$ value $=0.0487)(\mathbf{d})$. GSEA plot of PN21, upregulation of pyroptosis $(\mathrm{NES}=2.1968, p$ value $=0.0099)(\mathbf{e})$. f, g GSEA plots of PN35; upregulation of pyroptosis in PN35 $(\mathrm{NES}=1.9420, p$ value $=0.0087)(\mathbf{f})$; downregulation of protectors of tunicamycin induced ER stress (NES $=-1.3101, p$ value $=0.0608)$ (g). ${ }^{*}$ Custom gene sets of CRISPR screening library data. NES, normalised enrichment score (the larger the number, the greater the influence). PN, postnatal; CRISPR, clustered regularly interspaced short palindromic repeats; GSEA, gene set enrichment analysis; NES, normalised enrichment score; ER, endoplasmic reticulum.

(For figure see next page.) 

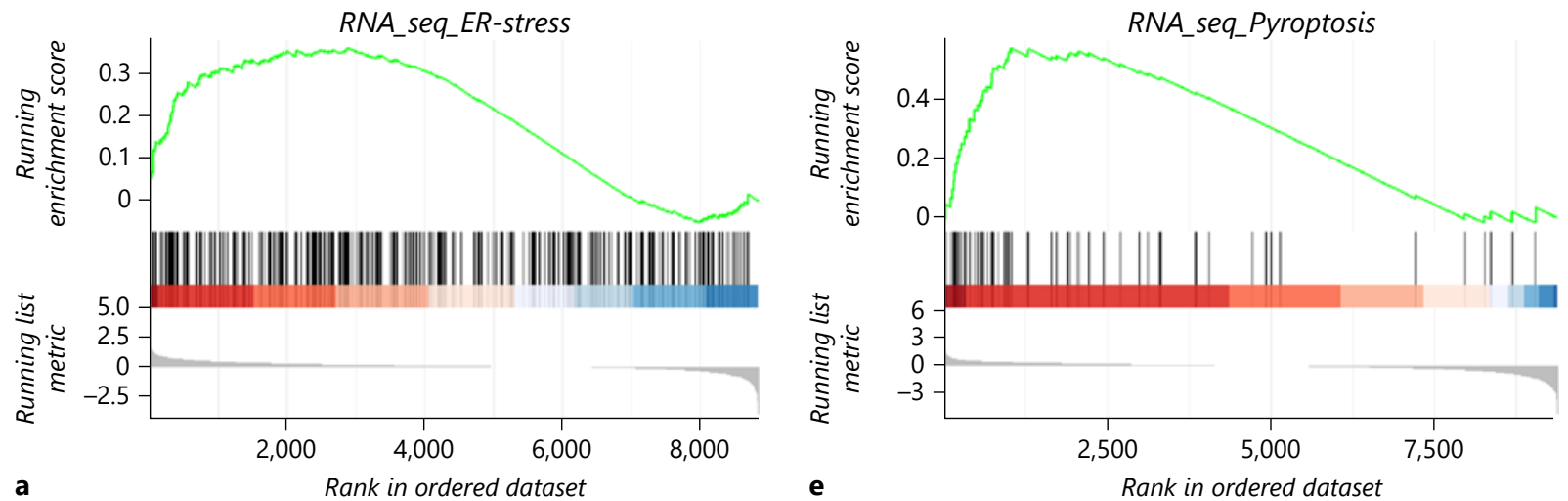

RNA_seq_Pyroptosis
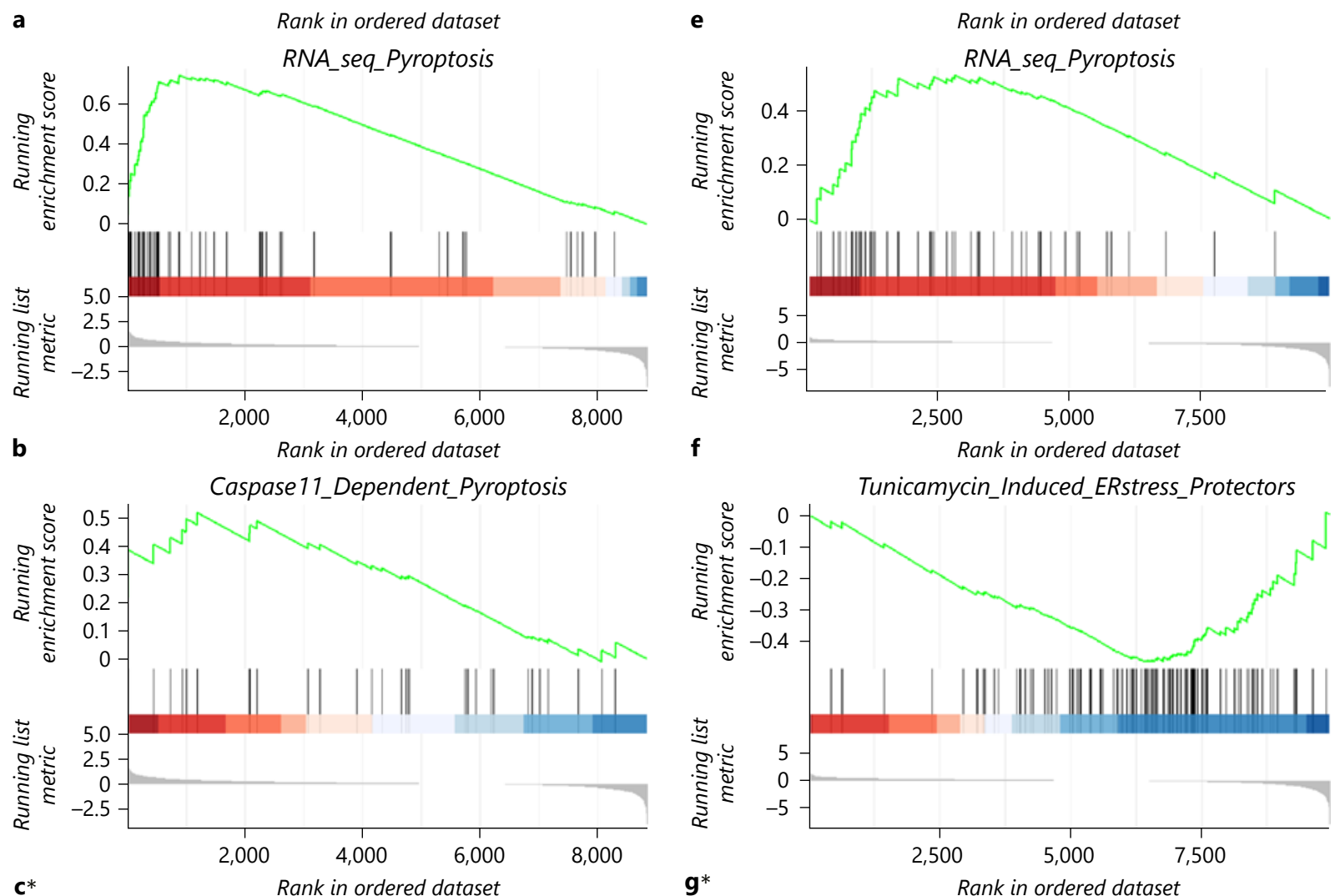

c*

$\mathrm{H}_{2} \mathrm{O}_{2}$ Induced Oxidative Stress_Sensitizers

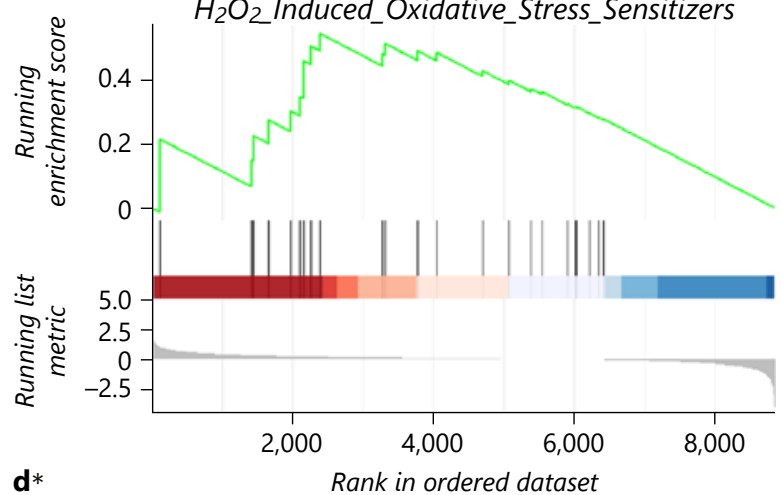




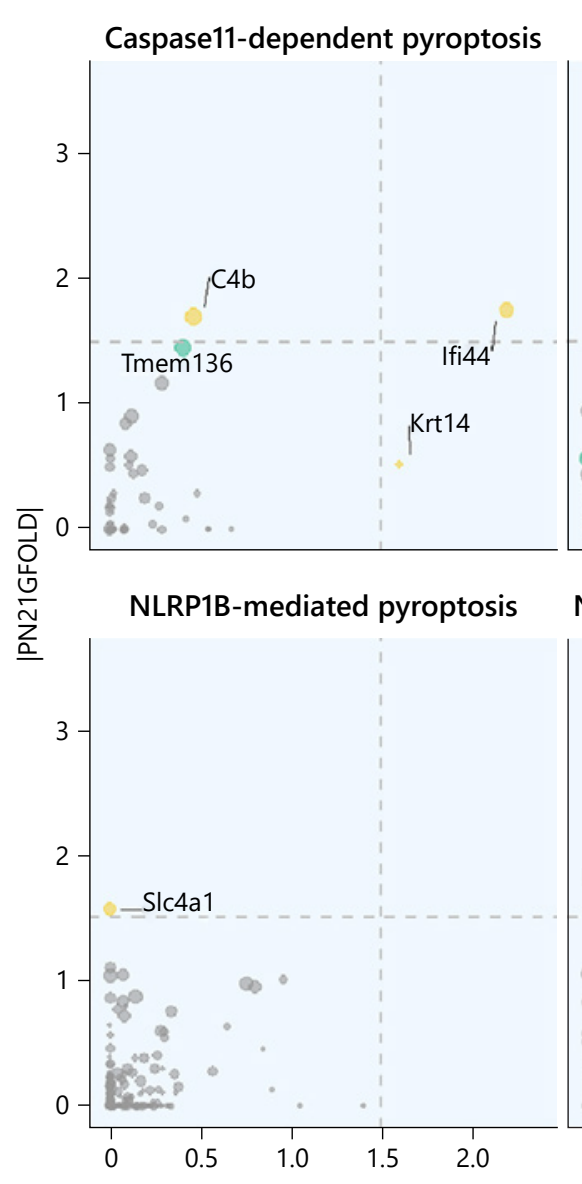

a

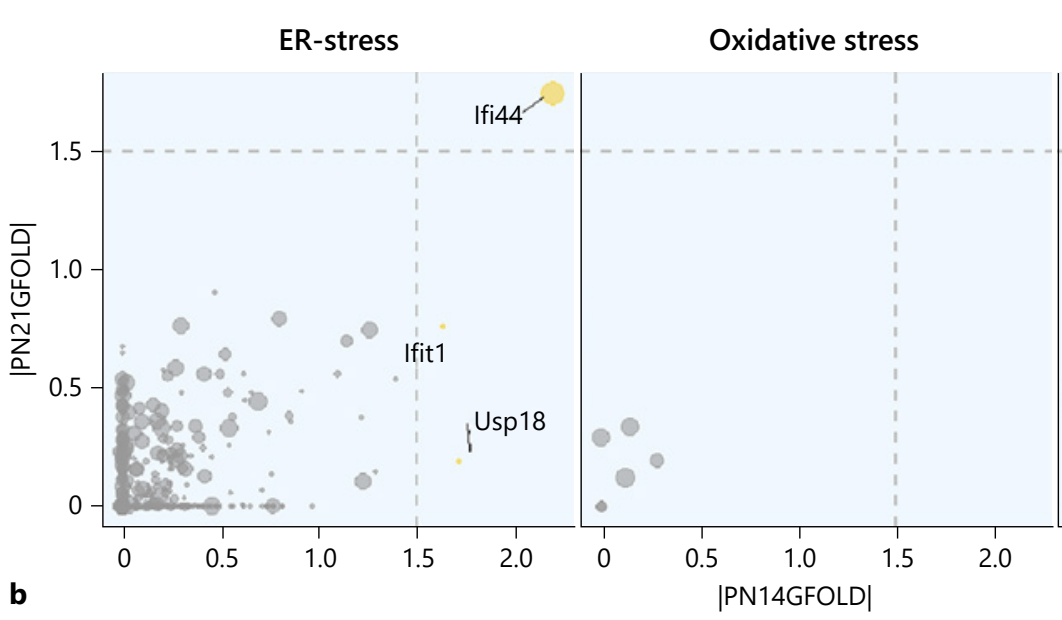

b

NRF2-related cellular homeostasis
$\mathrm{H}_{2} \mathrm{O}_{2}$-induced oxidative stress

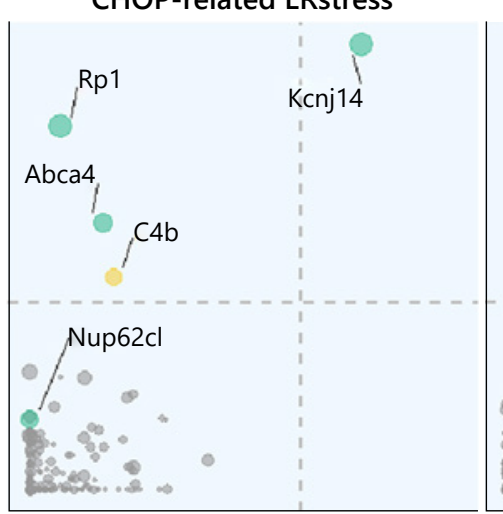

Tunicamycin-induced ER-stress

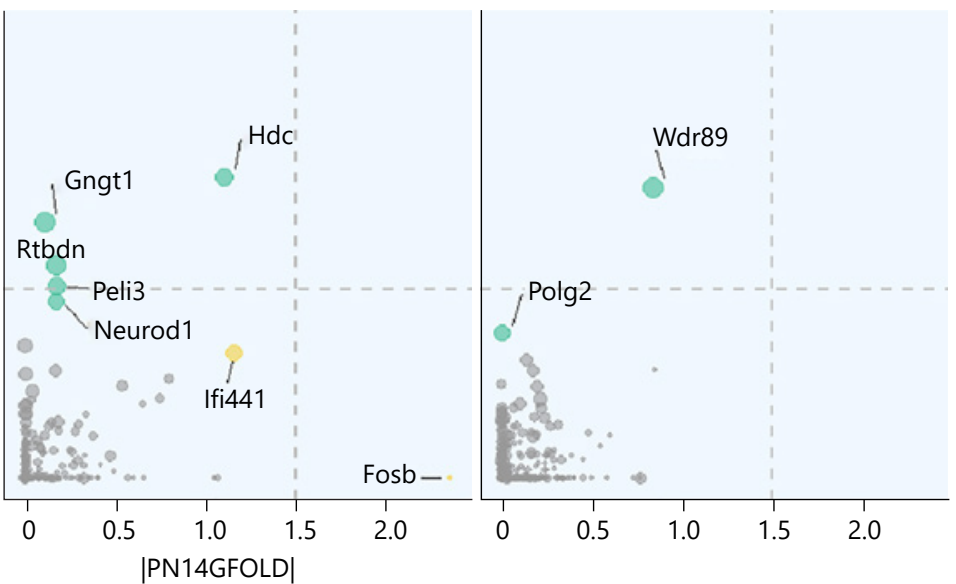

Pyroptosis

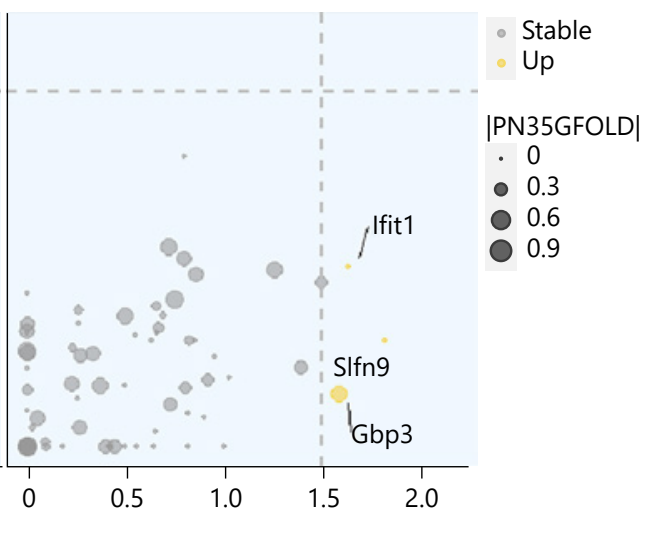

- Down

- Stable

- Up $\begin{array}{cc}\mid \text { PN35GFOLD| } \\ \cdot 0 \\ 0 & 1 \\ 0 & 2 \\ 0 & 3 \\ 0 & 4\end{array}$

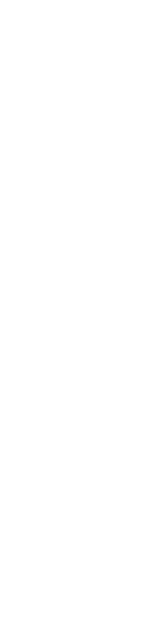



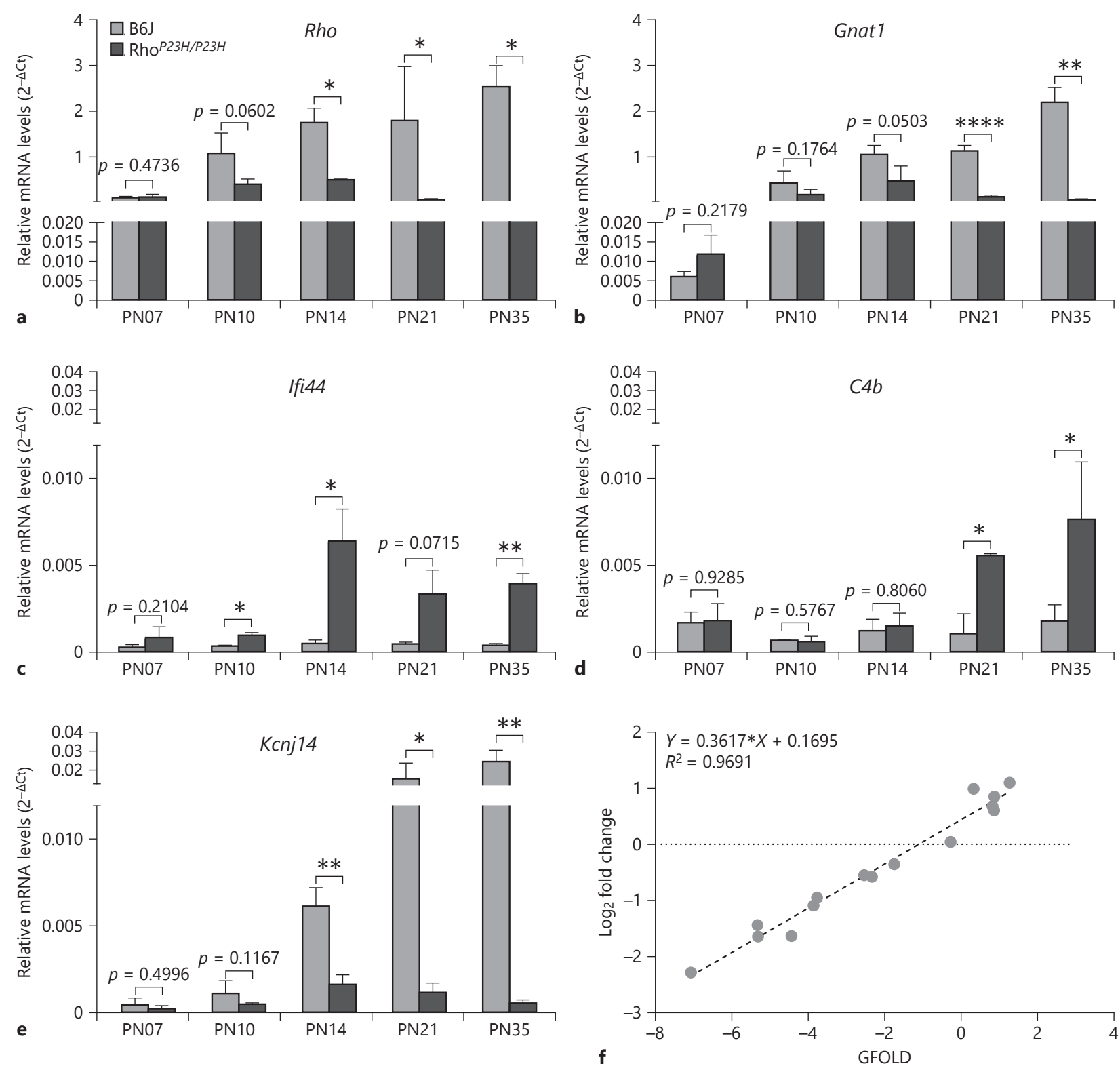

Fig. 4. Quantitative real-time PCR verification and correlation analysis between RNA-seq and qPCR data of selected genes. a-e Quantitative real-time PCR verification for Rho, Gnat1, Ifi44, $C 4 b$, and Kcnj14. Statistical analysis of the difference in the bar plots between $R h o^{P 23 H / P 23 H}$ mice and B6J mice at the same time point is performed with Student's $t$-test. ${ }^{*} p<0.05,{ }^{* *} p<0.01$,

${ }^{* * *} p<0.001,{ }^{* * * *} p<0.0001$. Biological replicates for each test $\geq 3$. f Correlation analysis between RNA-seq and qPCR data of selected genes at PN14, PN21, and PN35; Log2FoldChange was calculated using qPCR data. Linear regression model (red dotted line): $y=$ $0.3617 \mathrm{x}+0.1695, R^{2}=0.9691$. PN, postnatal; qPCR, quantitative PCR.

full row; this finding was consistent with previous research [3]. As for the functional analysis of the retina (online suppl. Fig. $1 j-s$ ), the fERG results show that the a-wave and b-wave amplitudes of $R h o^{P 23 H / P 23 H}$ mice were significantly smaller than those of B6J mice at PN21 and PN35 when

applying dark-adapted $3.0 \mathrm{~cd} \cdot \mathrm{s} / \mathrm{m}^{2}$ stimulus intensity. This response at dark-adapted $3.0 \mathrm{~cd} \cdot \mathrm{s} / \mathrm{m}^{2}$ is a mixed response from rod and cone photoreceptors. At PN21, from darkadapted $0.3-3 \mathrm{~cd} \cdot \mathrm{s} / \mathrm{m}^{2}$ and from light-adapted $1-10 \mathrm{~cd} \cdot \mathrm{s} /$ $\mathrm{m}^{2}$, an increase in the amplitudes of the a-wave and b-wave 
of $R h o^{P 23 H / P 23 H}$ mice was detected as the intensity of the stimulation increased. This indicates that parts of the cone photoreceptors are still able to respond to the light stimulus. However, at PN21 and PN35, there is no increase in the amplitudes of the a-wave and b-wave from dark-adapted $0.001-0.003 \mathrm{~cd} \cdot \mathrm{s} / \mathrm{m}^{2}$, implying rod photoreceptor dysfunction at this stage. Unfortunately, the fERG data at PN14 were missing because our instrument was unable to perform an accurate recording of flash electroretinogram at PN14 pups. Sufficient data are missing to determine the rod photoreceptor function of $R h o^{P 23 H / P 23 H}$ mice at an early stage. Our RNA-seq data and the results of the functional enrichment analysis are consistent with these phenotypic changes, which indicate the credibility of the RNA-seq data. A difference in the number of DEGs between our study and a recent study probably arose from different selection criteria and study design [35].

Considering the limitations and timeliness of the previous knowledge on enrichment analysis, we chose CRISPR screening library data for a more in-depth study. Genomewide CRISPR-Cas9 screening is a powerful technology that can link biological phenomenon to its corresponding genetic cause in mammalian cells through genome-wide mutagenesis [36], and it can be used to execute unbiased gene screening after applying selection pressure. We retrieved relevant articles for selecting CRISPR screening library data, and after exclusion of irrelevant data (most of the CRISPR screening library data are involved in the tumour, virus infection, and drug resistance), we finally got 6 CRISPR screening libraries, all associated with RP. Moreover, we also found some RNA-seq data for selection. Nevertheless, we found that these researches used different data processing software packages and screening criteria to process their data, so we aimed to use uniform standards to conduct evaluations for these data and relate them with our RNA-seq results. We next utilised GSEA to evaluate different mechanisms. The results of GSEA, together with the results of GO and KEGG analysis, provide important clues for the implications of the progression of $\mathrm{P} 23 \mathrm{H}$-related adRP.

The GSEA results show that PN14 is probably in peak inflammatory response as ER stress, pyroptosis, and oxidative stress are shown at PN14. A previous study showed that the misfolded rhodopsin accumulation due to $\mathrm{P} 23 \mathrm{H}$ opsin mutations occurred at an early stage of adRP [37]. Reactive oxygen species generated by misfolded proteins in the ER could induce the activation of the UPR [38], indicating that oxidative stress precedes ER stress. Cytoprotective and proapoptotic signalling coexisted in the initial stages of ER stress to cope with stress, and the early responses set in motion via IRE1 and ATF6 that attenuated if the stress per-

Exploring the Pathogenesis of $\mathrm{P} 23 \mathrm{H}-$

Related Retinitis Pigmentosa by RNA-Seq sisted, whereas PERK persisted under prolonged ER stress. Once these responses failed to re-establish homeostasis, proapoptotic signalling could lead photoreceptor cells towards apoptosis $[2,5,9]$. Meanwhile, reactive oxygen species could activate the NLRP3 inflammasome that links to neuroinflammation, such as pyroptosis $[6,39]$. Therefore, we infer that at PN14 oxidative stress may be activated by misfolded rhodopsin and subsequently induce both ER stress and pyroptosis. However, a previous study found only the upregulation of ER-associated degradation and no signal of other ER stress related changes when comparing the transcriptome of $\mathrm{P} 23 \mathrm{H}$ opsin expressed and wild-type opsin expressed in NIH3T3 cell lines [40]. This difference between this study and our results was probably due to differences between immortalised non-retinal cells and mouse photoreceptors. In another study, both ER-associated degradation and persistent ER stress could be observed in $\mathrm{P} 23 \mathrm{H}$ knock-in mice [3]. Downregulated phototransduction, together with pyroptosis, was observed in PN21. Moreover, in enrichment analysis at PN14 and PN21, the upregulation of intermediate filament-related pathways can be found. This result was consistent with a recent study performing RNA-seq of heterozygous $\mathrm{P} 23 \mathrm{H}$ mice, and the intermediate filament is associated with stress responses, apoptosis, and cell proliferation [41]. Furthermore, the analysis of RNAseq of heterozygous $\mathrm{P} 23 \mathrm{H}$ mice showed some retina adaption of scotopic vision loss. There were also no significant signs of cell death pathway function [35]. The above information indicates that animals at PN14 and PN21 undergo the process of photoreceptor death and retina cellular response. In PN35, persistent ER stress and pyroptosis still existed, whereas only less than a full row of photoreceptor nuclei was left, showing that these 2 mechanisms might be involved in other processes of retinal cell death. Interestingly, although the GSEA shows oxidative stress occurs at PN14, no DEGs that belonged to oxidative stress gene sets were observed at this time point. This result, together with the NES of different mechanisms, indicates that ER stress and pyroptosis play a significant role in the progression of P23H-related adRP.

After considering the intersection with custom gene sets made by CRISPR screening library and RNA-seq data, we obtained 22 candidate genes, 10 out of which have never been reported for their involvement in the retina (Ifi44, Ifi44l, Nup62 cl, Krt14, Tmem136, Slc4a1, Wdr89, Usp18, Gbp3, and Slfn9). There are 2 genes (Kcnj14 and Neurod1) enriched in the retina [42, 43], while 5 genes (Rp1, Abca4, Gngt1, Rtbdn, and $H d c$ ) were exclusively expressed in the photoreceptors. A total of 3 out of 5 (Rp1, Abca4, and Gngt1) were strongly associated 
with RP [44-48]. The aforementioned results show that some genes exclusively expressed in photoreceptors and enriched in the retina might be the critical genes in some mechanisms related to the inflammatory process. Even causing mutagenesis of these genes in non-retinal cells and then applying selection pressure can elicit the cellular response. The alterations of the expression levels of these genes in the process of some diseases may activate the mechanisms they are involved in. This finding can not only help our understanding of RP but also of other retinopathies in which these genes are involved.

Next, we chose 3 significant genes and subjected them to qPCR to verify the expression levels. For a complete timeline of the disease, we acquired 2 additional time points (PN07 and PN10) and used Rho and Gnat1 as references. The continually increasing expression levels between PN07 and PN35 of Rho and Gnat1 in B6J mice indicate that the retina is in the developmental stage. In the meantime, $R h o^{P 23 H / P 23 H}$ mice are in onset times; the expression levels do not reach the normal levels when they start to decrease. The trend of the expression level of Rho and Gnat1 could demonstrate the progression of RP at the RNA levels. In our candidate genes, Ifi44 and $C 4 b$ appeared concurrently in gene sets of ER stress and pyroptosis so that they might be in common molecular pathways of the 2 mechanisms, and their expression levels increase with the progression of the disease. The gene $C 4 b$ encodes complement factor 4 and involves complement activation and retinopathy such as diabetic retinopathy [49]. The gene Ifi44 encodes proliferative inhibitor, and it involves HCV infection and autoimmune diseases [50, 51]. Moreover, Ifi44 is involved in neurotoxin-induced glial-driven inflammation and neurodegeneration [52], and this connected Ifi44 with glial-driven inflammation, which is another factor contributing to RP. The gene Kcnj14 encodes Kir2.4, a rectifying potassium channel, which is abundant in the retina, especially dendritic tips of ON bipolar cells [42]; the decreasing rate of the expression level of Kcnj14 was faster than that of Gnat1, suggesting that the change in the expression level in Kcnj14 was related to early photoreceptor degeneration. Additionally, these 3 genes are also differentially expressed in heterozygous $\mathrm{P} 23 \mathrm{H}$ mice versus WT mice [35]. In summary, we conclude $C 4 b$, Ifi44, and Kcnj14 may play a role in the progression of RP, and further experiments could be conducted to explore whether these 3 genes are involved in or how they are involved.

There were several limitations to our study. (1) There are no biological replicates of our RNA-seq data, even though each sample has 6 retinas in it. To remedy this limitation, we use GFOLD, a reliable differential expres- sion tool, to provide a more biologically meaningful approach for RNA-seq data without biological replicates. Moreover, the results of the enrichment analysis match phenotypic changes. Correlation analysis shows the qPCR data are consistent with RNA-seq data. So, the aforementioned results show the credibility of our results. (2) The transformation between the mouse gene ID and human gene ID might miss small amounts of genes. Because of genomic differences between the 2 species, we cannot offer a way around this issue as of now. (3) We only performed the validations of RNA levels but not protein levels. The follow-up study is currently underway to make up for some of the shortcomings of this study.

In summary, we consider ER stress and pyroptosis to play the central role in the progression of RP, while oxidative stress could connect different mechanisms. Additionally, candidate genes such as Ifi44, C4b, and Kcnj14 deserve further study. Our findings offer some important clues for the study of RP.

\section{Statement of Ethics}

This study complied with the Association for Research in Vision and Ophthalmology Statement for the Use of Animals in Ophthalmic and Vision Research. Procedures were approved by the Institutional Animal Care and Use Committee of Peking University People's Hospital (permit number: 2016PHC059).

\section{Conflict of Interest Statement}

The authors have no conflicts of interest to declare.

\section{Funding Sources}

This work was supported by the National Natural Science Foundation of China (Grant Nos. 81600770, 81470649, and 81670870 ), the Science and Technology Innovation Project of the Chinese Academy of Medical Sciences (2019-RC-HL-019), and the Beijing Municipal Natural Science Foundation (Grant No. 7164306). The funders had no role in the study design, data collection and analysis, decision to publish, or preparation of the manuscript.

\section{Author Contributions}

Jiarui Li and Wei Du wrote this manuscript. Jiarui Li, Ningda $\mathrm{Xu}$, Tianchang Tao, and Xin Tang performed the experiment process. Jiarui Li conducted data processing and bioinformatic analysis. Wei Du and Lvzhen Huang provided the idea of this study and revised the manuscript. Jiarui $\mathrm{Li}$ and Wei Du have contributed equally to this work. 


\section{References}

1 Dryja TP, McGee TL, Reichel E, Hahn LB, Cowley GS, Yandell DW, et al. A point mutation of the rhodopsin gene in one form of retinitis pigmentosa. Nature. 1990;343(6256):364-6.

2 Lin JH, Li H, Yasumura D, Cohen HR, Zhang C, Panning B, et al. IRE1 signaling affects cell fate during the unfolded protein response. Science. 2007;318(5852):944-9.

3 Chiang WC, Kroeger H, Sakami S, Messah C, Yasumura D, Matthes MT, et al. Robust endoplasmic reticulum-associated degradation of rhodopsin precedes retinal degeneration. $\mathrm{Mol}$ Neurobiol. 2015;52(1):679-95.

4 Athanasiou D, Aguilà M, Bevilacqua D, Novoselov SS, Parfitt DA, Cheetham ME. The cell stress machinery and retinal degeneration. Febs Lett. 2013;587(13):2008-17.

5 Gorbatyuk MS, Knox T, LaVail MM, Gorbatyuk OS, Noorwez SM, Hauswirth WW, et al. Restoration of visual function in $\mathrm{P} 23 \mathrm{H}$ rhodopsin transgenic rats by gene delivery of BiP/Grp78. Proc Natl Acad Sci USA. 2010; 107(13):5961-6.

6 Viringipurampeer IA, Metcalfe AL, Bashar AE, Sivak O, Yanai A, Mohammadi Z, et al. NLRP3 inflammasome activation drives bystander cone photoreceptor cell death in a $\mathrm{P} 23 \mathrm{H}$ rhodopsin model of retinal degeneration. Hum Mol Genet. 2016;25(8):1501-16.

7 Sanvicens N, Gómez-Vicente V, Masip I, Messeguer A, Cotter TG. Oxidative stress-induced apoptosis in retinal photoreceptor cells is mediated by calpains and caspases and blocked by the oxygen radical scavenger CR6. J Biol Chem. 2004;279(38):39268-78.

8 Wen RH, Stanar P, Tam B, Moritz OL. Autophagy in xenopus laevis rod photoreceptors is independently regulated by phototransduction and misfolded RHOP23H. Autophagy. 2019;15(11):1970-89.

9 Comitato A, Schiroli D, Montanari M, Marigo V. Calpain activation is the major cause of cell death in photoreceptors expressing a rhodopsin misfolding mutation. Mol Neurobiol. 2020;57(2):589-99.

10 Adekeye A, Haeri M, Solessio E, Knox BE. Ablation of the proapoptotic genes chop or ask1 does not prevent or delay loss of visual function in a $\mathrm{P} 23 \mathrm{H}$ transgenic mouse model of retinitis pigmentosa. Plos One. 2014;9(2): e83871.

11 Di Pierdomenico J, García-Ayuso D, AgudoBarriuso M, Vidal-Sanz M, Villegas-Pérez MP. Role of microglial cells in photoreceptor degeneration. Neural Regen Res. 2019;14(7): 1186-90.

12 Sakami S, Imanishi Y, Palczewski K. Müller glia phagocytose dead photoreceptor cells in a mouse model of retinal degenerative disease. FASEB J. 2019;33(3):3680-92.

13 Athanasiou D, Aguila M, Bellingham J, Li W, McCulley C, Reeves PJ, et al. The molecular and cellular basis of rhodopsin retinitis pigmentosa reveals potential strategies for therapy. Prog Retin Eye Res. 2018;62:1-23.
14 Sakami S, Maeda T, Bereta G, Okano K, Golczak M, Sumaroka A, et al. Probing mechanisms of photoreceptor degeneration in a new mouse model of the common form of autosomal dominant retinitis pigmentosa due to P23H opsin mutations. J Biol Chem. 2011; 286(12):10551-67.

15 Liao Y, Smyth GK, Shi W. Feature counts: an efficient general purpose program for assigning sequence reads to genomic features. Bioinformatics. 2014;30(7):923-30.

16 Feng J, Meyer CA, Wang Q, Liu JS, Shirley Liu $X$, Zhang Y. GFOLD: a generalized fold change for ranking differentially expressed genes from RNA-seq data. Bioinformatics. 2012;28(21):2782-8.

17 Szklarczyk D, Gable AL, Lyon D, Junge A, Wyder S, Huerta-Cepas J, et al. STRING v11: protein-protein association networks with increased coverage, supporting functional discovery in genome-wide experimental datasets. Nucleic Acids Res. 2019;47(D1):D607D13.

18 Yu G, Wang LG, Han Y, He QY. Cluster profiler: an $\mathrm{R}$ package for comparing biological themes among gene clusters. Omics. 2012; 16(5):284-7.

19 Durinck S, Spellman PT, Birney E, Huber W. Mapping identifiers for the integration of genomic datasets with the R/bioconductor package biomaRt. Nat Protoc. 2009;4(8): 1184-91.

20 Schmittgen TD, Livak KJ. Analyzing realtime PCR data by the comparative C (T) method. Nat Protoc. 2008;3(6):1101-8.

21 Aguila M, Bevilacqua D, McCulley C, Schwarz N, Athanasiou D, Kanuga N, et al. Hsp90 inhibition protects against inherited retinal degeneration. Hum Mol Genet. 2014;23(8): 2164-75.

22 Spandidos A, Wang X, Wang H. Seed B. PrimerBank: a resource of human and mouse PCR primer pairs for gene expression detection and quantification. Nucleic Acids Res. 2010;38(Database issue):D792-9.

23 Hasan M, Seo JE, Rahaman KA, Min H, Kim $\mathrm{KH}$, Park JH, et al. Novel genes in brain tissues of EAE-induced normal and obese mice: upregulation of metal ion-binding protein genes in obese-EAE mice. Neuroscience. 2017;343:322-36.

24 Thomas KC, Zheng XF, Garces Suarez F, Raftery JM, Quinlan KG, Yang N, et al. Evidence based selection of commonly used RT-qPCR reference genes for the analysis of mouse skeletal muscle. Plos One. 2014;9(2):e88653.

25 Charish J, Shabanzadeh AP, Chen D, Mehlen $\mathrm{P}$, Sethuramanujam S, Harada $\mathrm{H}$, et al. Neogenin neutralization prevents photoreceptor loss in inherited retinal degeneration. J Clin Invest. 2020;130(4):2054-68.

26 Dubreuil MM, Morgens DW, Okumoto K, Honsho M, Contrepois K, Lee-McMullen B, et al. Systematic identification of regulators of oxidative stress reveals non-canonical roles for peroxisomal import and the pentose phosphate pathway. Cell Rep. 2020;30(5):1417-e7.

27 Schinzel RT, Higuchi-Sanabria R, Shalem O, Moehle EA, Webster BM, Joe L, et al. The hyaluronidase, TMEM2, promotes ER homeostasis and longevity independent of the UPR(ER). Cell. 2019;179(6):1306-18 e18.

28 Panganiban RA, Park HR, Sun M, Shumyatcher M, Himes BE, Lu Q. Genome-wide CRISPR screen identifies suppressors of endoplasmic reticulum stress-induced apoptosis. Proc Natl Acad Sci U S A. 2019;116(27):13384-93.

29 Kerins MJ, Liu P, Tian W, Mannheim W, Zhang DD, Ooi A. Genome-wide CRISPR screen reveals autophagy disruption as the convergence mechanism that regulates the NRF2 transcription factor. Mol Cell Biol. 2019;39(13):e00037-19.

30 Napier BA, Brubaker SW, Sweeney TE, Monette P, Rothmeier GH, Gertsvolf NA, et al. Complement pathway amplifies caspase11-dependent cell death and endotoxin-induced sepsis severity. J Exp Med. 2016; 213(11):2365-82.

31 Chui AJ, Okondo MC, Rao SD, Gai K, Griswold AR, Johnson DC, et al. N-terminal degradation activates the NLRP1B inflammasome. Science. 2019;364(6435):82-5.

32 Zhang C, Zhao C, Chen X, Tao R, Wang S, Meng G, et al. Induction of ASC pyroptosis requires gasdermin $\mathrm{D}$ or caspase-1/11-dependent mediators and IFN $\beta$ from pyroptotic macrophages. Cell Death Dis. 2020;11(6):470.

33 Han J, Back SH, Hur J, Lin YH, Gildersleeve R, Shan J, et al. ER-stress-induced transcriptional regulation increases protein synthesis leading to cell death. Nat Cell Biol. 2013;15(5):481-90.

34 Sastry KS, Naeem H, Mokrab Y, Chouchane AI. RNA-seq reveals dysregulation of novel melanocyte genes upon oxidative stress: implications in vitiligo pathogenesis. Oxid Med Cell Longev. 2019;2019:2841814.

35 Leinonen H, Pham NC, Boyd T, Santoso J, Palczewski K, Vinberg F. Homeostatic plasticity in the retina is associated with maintenance of night vision during retinal degenerative disease. Elife. 2020;9:e59422.

36 Yu JSL, Yusa K. Genome-wide CRISPR-Cas9 screening in mammalian cells. Methods. 2019;165:29-35.

37 Saliba RS, Munro PM, Luthert PJ, Cheetham ME. The cellular fate of mutant rhodopsin: quality control, degradation and aggresome formation. J Cell Sci. 2002;115(Pt 14):2907-18.

38 Malhotra JD, Miao H, Zhang K, Wolfson A, Pennathur S, Pipe SW, et al. Antioxidants reduce endoplasmic reticulum stress and improve protein secretion. Proc Natl Acad Sci USA. 2008;105(47):18525-30.

39 Wei P, Yang F, Zheng Q, Tang W, Li J. The potential role of the NLRP3 inflammasome activation as a link between mitochondria ROS generation and neuroinflammation in postoperative cognitive dysfunction. Front Cell Neurosci. 2019;13:73.
Exploring the Pathogenesis of $\mathrm{P} 23 \mathrm{H}-$ Related Retinitis Pigmentosa by RNA-Seq
Ophthalmic Res 2021;64:798-810 DOI: $10.1159 / 000515727$ 
40 Chen Y, Brooks MJ, Gieser L, Swaroop A, Palczewski K. Transcriptome profiling of NIH3T3 cell lines expressing opsin and the $\mathrm{P} 23 \mathrm{H}$ opsin mutant identifies candidate drugs for the treatment of retinitis pigmentosa. Pharmacol Res. 2017;115:1-13.

41 Nishimura Y, Kasahara K, Inagaki M. Intermediate filaments and IF-associated proteins: from cell architecture to cell proliferation. Proc Jpn Acad Ser B Phys Biol Sci. 2019;95(8): 479-93.

42 Dhingra A, Sulaiman P, Xu Y, Fina ME, Veh RW, Vardi N. Probing neurochemical structure and function of retinal $\mathrm{ON}$ bipolar cells with a transgenic mouse. J Comp Neurol. 2008;510(5):484-96.

43 Pennesi ME, Cho JH, Yang Z, Wu SH, Zhang $\mathrm{J}, \mathrm{Wu} \mathrm{SM}$, et al. BETA2/NeuroD1 null mice: a new model for transcription factor-dependent photoreceptor degeneration. J Neurosci. 2003;23(2):453-61.
44 Yamashita T, Liu J, Gao J, LeNoue S, Wang C, Kaminoh J, et al. Essential and synergistic roles of RP1 and RP1L1 in rod photoreceptor axoneme and retinitis pigmentosa. J Neurosci. 2009;29(31):9748-60.

45 Cremers FP, van de Pol DJ, van Driel M, den Hollander AI, van Haren FJ, Knoers NV, et al. Autosomal recessive retinitis pigmentosa and cone-rod dystrophy caused by splice site mutations in the Stargardt's disease gene ABCR. Hum Mol Genet. 1998;7(3):355-62.

46 Scherer SW, Feinstein DS, Oliveira L, Tsui LC, Pittler SJ. Gene structure and chromosome localization to $7 \mathrm{q} 21.3$ of the human rod photoreceptor transducin gamma-subunit gene (GNGT1). Genomics. 1996;35(1):241-3.

47 Kelley RA, Al-Ubaidi MR, Naash MI. Retbindin is an extracellular riboflavin-binding protein found at the photoreceptor/retinal pigment epithelium interface. J Biol Chem. 2015; 290(8):5041-52.

48 Greferath U, Vessey KA, Jobling AI, Mills SA, Bui BV, He Z, et al. The role of histamine in the retina: studies on the Hdc knockout mouse. PLoS One. 2014;9(12):e116025.
49 Shahulhameed S, Vishwakarma S, Chhablani J, Tyagi M, Pappuru RR, Jakati S, et al. A systematic investigation on complement pathway activation in diabetic retinopathy. Front Immunol. 2020;11:154.

50 Hallen LC, Burki Y, Ebeling M, Broger C, Siegrist F, Oroszlan-Szovik K, et al. Antiproliferative activity of the human IFN-alpha-inducible protein IFI44. J Interferon Cytokine Res. 2007;27(8):675-80.

51 Brkic Z, Maria NI, van Helden-Meeuwsen CG, van de Merwe JP, van Daele PL, Dalm VA, et al. Prevalence of interferon type I signature in CD14 monocytes of patients with Sjogren's syndrome and association with disease activity and BAFF gene expression. Ann Rheum Dis. 2013;72(5):728-35.

52 Pachiappan A, Thwin MM, Manikandan J, Gopalakrishnakone P. Glial inflammation and neurodegeneration induced by candoxin, a novel neurotoxin from Bungarus candidus venom: global gene expression analysis using microarray. Toxicon. 2005;46(8):883-99. 